INPLASY

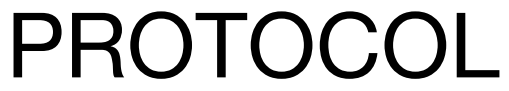

To cite: Wei et al. Risk factors for emergence agitation in adults after general anesthesia: A meta-analysis from observational studies. Inplasy protocol 202070032. doi:

10.37766/inplasy2020.7.0032

Received: 10 July 2020

Published: 10 July 2020

Corresponding author: Bing Chen

chenbing2050@163.com

Author Affiliation:

The Second Affiliated Hospital of Chongqing Medica

Support: None.

Review Stage at time of this submission: Preliminary searches.

Conflicts of interest: None.

\section{Risk factors for emergence agitation in adults after general anesthesia: A meta-analysis from observational studies}

Wei, B1; Feng, Y2; Chen, W3; Ren, D4; Xiao, D5; Chen, B6.

Review question / Objective: This meta-analysis aims to find out the preoperative and perioperative factors for emergence agitation in adults after general anesthesia based on observational studies. (1) Patients: adult patients intend to extubation and emergence at operation room, postanesthesia care unit or intensive care unit after general anesthesia. (2) Intervention or exposure: diagnosed as emergence agitation according to the predefined scale. (3) Comparator or control: diagnosed as non-emergence agitation according to the predefined scale. (4) Outcomes: the risk ratio and mean difference of risk factors, including age, male, ASA grade, BMI, education, smoking, drinking, pre-existing of illness, preexisting use of drugs, history of surgery, perioperative use of drugs, types of anesthesia and airway, length of anesthesia, bleeding volume and transfusion, fluid intake, body temperature, types of surgery, urinary catheter, gastric tube and postoperative pain. (5) Study design: observational studies, cohort studies, case-control studies.

INPLASY registration number: This protocol was registered with the International Platform of Registered Systematic Review and Meta-Analysis Protocols (INPLASY) on 10 July 2020 and was last updated on 10 July 2020 (registration number INPLASY202070032).

\section{INTRODUCTION}

Review question / Objective: This metaanalysis aims to find out the preoperative and perioperative factors for emergence agitation in adults after general anesthesia based on observational studies. (1) Patients: adult patients intend to extubation and emergence at operation room, postanesthesia care unit or intensive 
care unit after general anesthesia. (2) Intervention or exposure: diagnosed as emergence agitation according to the predefined scale. (3) Comparator or control: diagnosed as non-emergence agitation according to the predefined scale. (4) Outcomes: the risk ratio and mean difference of risk factors, including age, male, ASA grade, BMI, education, smoking, drinking, pre-existing of illness, preexisting use of drugs, history of surgery, perioperative use of drugs, types of anesthesia and airway, length of anesthesia, bleeding volume and transfusion, fluid intake, body temperature, types of surgery, urinary catheter, gastric tube and postoperative pain. (5) Study design: observational studies, cohort studies, case-control studies.

Condition being studied: Emergence agitation, is characterized by agitation, restlessness and hyperactivity during the transition from unconsciousness to full wakefulness, therefore, it also names emergence agitation or emergence excitement or emergence delirium. Emergence agitation is an adverse postoperative complication that can occur in patients of any age, but most often in children with a variable incidence of 10$80 \%$ and elderly patients between $10 \%$ and $15 \%$. In the adult population, ED incidence was reported differently from 4.7 to $22.2 \%$. Emergence agitation increases the risk for injury, self-extubation, hemorrhages and prolonged hospitalization. Risk factors for pediatric emergence agitation are the child's age, genetic profile, length and type of surgical procedure, and use of inhalational anesthesia. However, the risk factors in adults are unclear.

\section{METHODS}

Search strategy: Pubmed \#1 delirium[TIAB] OR agitation [TIAB] OR inadequate[TIAB] OR excitement[TIAB] \#2 emergence[TIAB] \#3 observational[all fields] OR cohort [all fields] OR case-control[all fields] OR case control[all fields] \#4 \#1 AND \#2 AND \#3. Medline \#1 delirium[TS] OR agitation [TS] OR inadequate[TS] OR excitement[TS] \#2 emergence[TS] \#3 observational[TS] OR cohort [TS] OR case-control[TS] OR case control[TS] \#4 \#1 AND \#2 AND \#3. Embase and Cochrane library \#1 delirium[ti,ab,kw] OR agitation [ti, ab,kw] OR in adequate [ $t i, a b, k w]$ OR excitement [ $t i, a b, k w] \quad 2$ emergence[ti,ab,kw]\#3 observational[all fields] OR cohort [all fields] OR casecontrol[all fields] OR case control[all fields] \#4 \#1 AND \#2 AND \#3.

Participant or population: Adult patients intend to extubation and emergence at operation room, postanesthesia care unit or intensive care unit after general anesthesia.

Intervention: Diagnosed as emergence agitation according to the predefined scale.

Comparator: Diagnosed as non-emergence agitation according to the predefined scale.

Study designs to be included: Observational studies, cohort studies, case-control studies.

Eligibility criteria: (1) Patients: adult patients intend to extubation and emergence at operation room, postanesthesia care unit or intensive care unit after general anesthesia. (2) Exposure: diagnosed as emergence agitation according to the predefined scale. (3) Control: diagnosed as non-emergence agitation according to the predefined scale.

(4) Outcomes: reported the number of patients or mean difference for preoperative and periopreative risk factors in agitated and non-agitated groups. (5) Study design: observational studies, cohort studies, case-control studies.

Information sources: Electronic databases: Embase (1980-2020.06), PubMed (19662020.06), Medline (1966-2020.06) and the Cochrane Library. Any potentially relevant trials will be manually searched based on the references of the identified trials and systematic reviews.

Main outcome(s): The risk ratio of risk factors for emergence agitation, these risk factors including preoperative and 
perioperative factors. The integrated risk ratio will be calculated when there are two studies reported the same risk factor. Considering there is no unified diagnostic criteria for emergence agitation, the number of emergence agitation will be directly extracted from the literature. The dichotomous data of risk factors will be extracted and integrated to calculate the risk ratio.

Additional outcome(s): The mean difference of risk factors for emergence agitation. Some data of risk factors were continuous data, these will be meta-analyzed to strength the dichotomous data. The continuous data of risk factors will be extracted and integrated to calculate the mean difference.

Quality assessment / Risk of bias analysis: The quality of observational studies will be evaluated using the Newcastle-Ottawa Scale (NOS) with a maximum total score of 9 that will be judged at population selection, comparability and outcomes.

Strategy of data synthesis: Review manager software 5.4 will be used for meta-analysis. Mean difference (MD) with 95\% confidence intervals (Cls) will be used to assess continuous outcomes. Risk ratio (RR) with $95 \%$ Cls will be used to assess dichotomous outcomes. The meta-analysis was conducted when two or more trials reported similar outcomes. Statistical heterogeneity among included studies will be assessed by Chi2 (P) and I2. Because this meta-analysis is based on observational studies, thus, a randomeffects model will be applied. The inverse variance and Mantel-Haenszel methods will be used to combine separate statistics. $P$ values less than 0.05 is considered significant difference.

Subgroup analysis: If statistical heterogeneity is significant ( $\mathrm{P}$ below 0.1 or $I^{2}$ above $50 \%$ ), a subgroup analysis will be performed to investigate the source of heterogeneity. We will use the following subgroup hypotheses to explain heterogeneity among studies: 1) different diagnostic criteria for emergence agitation; 2) studies were of high-quality.

Sensibility analysis: Sensitivity analysis will be conducted by omitting one study in turn.

Language: English.

Country(ies) involved: China.

Keywords: Risk factor; adults; emergence agitation; emergence delirium; metaanalysis.

Contributions of each author:

Author 1 - Bing Wei - Author 1 collected and analyzed the data, generated figures and wrote the manuscript.

Author 2 - Yan Feng - The author collected and analyzed the data, generated figures and wrote the manuscript.

Author 3 - Wen Chen - The author collected and analyzed the data.

Author 4 - Da Ren - The author revised the draft, data and figures.

Author 5 - Dai Xiao - The author revised the draft, data and figures, wrote and proofread the manuscript.

Author 6 - Bing Chen - The author designed the study, wrote and proofread the manuscript. 